\title{
MEDICAID EXPANSIONS \\ AND THE CROWDING OUT \\ OF PRIVATE HEALTH INSURANCE
}

Esel Y. Yazici

Robert Kaestner

Working Paper 6527 


\title{
MEDICAID EXPANSIONS \\ AND THE CROWDING OUT \\ OF PRIVATE HEALTH INSURANCE
}

Esel Y. Yazici

Robert Kaestner

Working Paper 6527

http://www.nber.org/papers/w6527

\section{NATIONAL BUREAU OF ECONOMIC RESEARCH \\ 1050 Massachusetts Avenue \\ Cambridge, MA 02138 \\ April 1998}

\begin{abstract}
Any opinions expressed are those of the authors and not those of the National Bureau of Economic Research.

(C) 1998 by Esel Y. Yazici and Robert Kaestner. All rights reserved. Short sections of text, not to exceed two paragraphs, may be quoted without explicit permission provided that full credit, including (C) notice, is given to the source.
\end{abstract}


Medicaid Expansions and the Crowding Out

of Private Health Insurance

Esel Y. Yazici and Robert Kaestner

NBER Working Paper No. 6527

April 1998

JEL Nos. I38, H42

\begin{abstract}
In this paper, we re-examine the question of crowd out among children. Our primary contribution is the use of longitudinal data. These data allow us to identify several groups of children depending on whether their eligibility for Medicaid was affected by the eligibility expansions, and to investigate whether changes in insurance coverage of children affected by the expansions differed from changes in insurance coverage of children unaffected by the expansions. For example, we directly measure whether children who became eligible for Medicaid due to the expansions decreased their enrollment in private insurance plans faster than children whose eligibility for Medicaid was unaffected by the expansions. Our results suggest that there was relatively little crowd out among children. We estimate that 14.5 percent of the recent increase in Medicaid enrollment came from private insurance.
\end{abstract}

Esel Y. Yazici

Bane, Cueno and Associates

BJK Plaza Spor Cad. A Blok No: 125-126

Besiktas 81180 Istanbul

TURKEY
Robert Kaestner

National Bureau of Economic Research

50 East 42nd Street

17th Floor

New York, NY 10017-5405

Rjkbb@cunyvm.cuny.edu 


\section{Introduction}

Starting in the mid-1980s, Congress expanded Medicaid coverage to near-poor pregnant women and children in an attempt to reduce infant mortality and improve infant and child health. Beginning with the Deficit Reduction Act of 1984, the link between Medicaid eligibility and other cash assistance programs was severed, and low-income pregnant women and children who did not fit into traditional welfare categories gained access to publicly financed health care. As a result, from 1988 to 1992 the percentage of children under eighteen enrolled in Medicaid rose from 15.6 percent to 21.6 percent. At the same time, the number of uninsured children declined only slightly and a substantial decrease occurred in the number of children covered by private insurance (Newacheck et al. 1995). These trends suggest that part of the gains in Medicaid enrollment may have been due to crowding out of private insurance.

The extent of crowd out is an important public policy issue because it reduces the effectiveness of government expenditures. Every dollar that unintentionally goes toward subsidizing previously insured persons becomes unavailable to be used to reduce the number of uninsured, the intended target group of the expansions. Interest in the issue of crowd out continues to be strong since previous studies of the problem have reached dissimilar conclusions about the magnitude of crowd out. Cutler and Gruber (1996) found that between 30 to 40 percent of the increased enrollment in Medicaid among children came at the expense of private insurance, while Shore-Sheppard (1997) and Dubay and Kenney (1996a) reported a much smaller crowd out effect of between 15 and 22 percent for children. Similar differences are found among estimates of crowd out for women. Cutler and Gruber (1996) concluded that 50 percent of the increased enrollment in Medicaid among women consisted of switches from private insurance to Medicaid. In contrast, Dubay and Kenney (1996b) estimated a crowd out effect of only 15 percent for pregnant women. The mixed nature of these findings leaves the question of the true extent of crowd out unresolved and justifies additional study. Indeed, the amount of crowd out was an intensely contested issue in recent debates over the expansion of publicly financed health insurance to near-poor children contained in the Balanced Budget Act of 1997.

In this paper, we re-examine the question of crowd out among children. Our primary contribution to this literature is the use of longitudinal data. These data allow us to identify several groups of children depending on 
whether their eligibility for Medicaid was affected by the eligibility expansions, and to investigate whether changes in insurance coverage of children affected by the expansions differed from changes in insurance coverage of children unaffected by the expansions. For example, we can directly measure whether children who became eligible for Medicaid due to the expansions decreased their enrollment in private insurance plans faster than children whose eligibility for Medicaid was unaffected by the expansions. Previous studies have relied on repeat cross-sectional data from the Current Population Survey (CPS) that precluded the direct observation of changes in eligibility status or changes in insurance coverage. As a result, these studies had to infer the effects of the Medicaid eligibility expansions from cross-sectional and time variation in the distribution of insurance coverage that was associated with Medicaid eligibility.

\section{Past Research}

Three recent studies have investigated the effects of Medicaid eligibility expansions on the health insurance status of children using pooled cross-sectional data from the Current Population Survey (CPS). The most widely cited study is by Cutler and Gruber (1996) who used a relatively simple regression procedure to estimate the effect of Medicaid eligibility on insurance status (Medicaid, private insurance, or uninsured). They control for a variety of personal characteristics and include year and state dummy variables in the model to control for state and time effects. Their results indicated that 30 to 40 percent of the increase in Medicaid enrollment of children between 1988 and 1993 was a due to a reduction in private insurance coverage of children.

The Cutler and Gruber (1996) study has two potential weaknesses. The first is related to their sample and the way in which they control for unmeasured state and time variation in insurance status. Cutler and Gruber (1996) included all children in their analysis and restrict state and time effects to be equal for children who were eligible for Medicaid and those who were ineligible for Medicaid. This strategy may be problematic if state and time effects differ by Medicaid eligibility status. Indeed, Acs and Steurle (1993) show that changes in health insurance coverage of the Medicaid-eligible (i.e., low income) population are different than those of the Medicaid ineligible (i.e., high-income) population. This problem is significant because Cutler and Gruber (1996) do not actually observe insurance status switches, but estimate the amount of switching from changes over time within 
insurance status. Their regression estimates of crowd out are obtained under the assumption that deviations from trend in private insurance that are associated with Medicaid eligibility represent causal switches between private insurance and Medicaid. If trends in private insurance differ by Medicaid eligibility status, Cutler and Gruber's estimates of the effect of Medicaid eligibility on private insurance will be biased. Another way of stating this is that Cutler and Gruber (1996) may have used an inappropriate control group to obtain the effect of Medicaid eligibility on insurance coverage. In their case, the time and state dummy variables serve as the control group and measure state and time variation in insurance status.

The second potential problem with the Cutler and Gruber (1996) study is that their estimates may be biased because of measurement error associated with Medicaid eligibility. Medicaid eligibility is calculated using reported past-calendar year income, which may be a poor proxy of current income, particularly for low-income families who typically have greater income variability than high-income families. This suggests that there will be some degree of measurement error and that measurement error in eligibility is non-random since low-income families are also more likely to be eligible for Medicaid. The non-random nature of the measurement error results in biased estimates with the sign of the bias unknown. Moreover, the measurement error problem may be severe because of the shape of the distribution of family incomes and the discrete nature of the Medicaid eligibility assignment rule. For a significant majority of families during the period of the Cutler and Gruber (1996) analysis, their incomes were well above the income eligibility threshold, and it is unlikely that measurement error affected their eligibility assignment. Consequently, a majority of families in the sample whose incomes are relatively high and who have a low probability of Medicaid eligibility are likely to be correctly assigned eligibility and have zero measurement error. On the other hand, low-income families with a high probability of being eligible for Medicaid will be more likely to be incorrectly assigned eligibility because their incomes are closer to the eligibility threshold. Consequently, measurement error and true Medicaid eligibility are positively correlated, reinforcing the expectation that the measurement error in eligibility is non-random. ${ }^{1}$

\footnotetext{
${ }^{1}$ Note that in a simple one regressor model, the positive correlation implies that the estimated parameter will be larger than the true parameter.
} 
The extent of measurement error in Medicaid eligibility is difficult to determine since information about the number of ineligible children who are incorrectly assigned is not available. We do, however, have information on how many children in the CPS who were classified as ineligible were actually covered by Medicaid. Dubay and Kenney (1996a), who also use the CPS, reported (Table 2) that Medicaid covered approximately 20.0 percent of ineligible low-income children in their sample. Similarly, Shore-Sheppard (1995) reported that in 1988, Medicaid covered 3.3 percent of all ineligible children. The 3.3 percent figure does not seem large until one realizes that it represents 80 percent of all non-AFDC eligible Medicaid recipients. Finally, Shore-Sheppard (1995) presented evidence that measurement error associated with Medicaid eligibility is growing over time as true eligibility increases. In 1988, 3.3 percent of ineligible children reported Medicaid coverage, but by 1993, 5.4 percent of ineligible children reported Medicaid coverage.

We also contend that the instrumental variables procedure used by Cutler and Gruber (1996) may not have adequately addressed the measurement error problem. Cutler and Gruber (1996) instrument for measured eligibility of children in a state using an instrument they refer to as "simulated eligibility", which is the average eligibility of a national sample of children determined by applying that state's eligibility rules to the national sample. The measurement error problem, however, is also present in the national sample since income is poorly measured for this group. Thus, the "simulated eligibility" measure consists of the average true eligibility and an average error. In states with a relatively low Medicaid income eligibility threshold, the average measurement error for the national sample will tend to be small since most families' incomes in the national sample are well above the threshold. The average measurement error in the national sample will also tend to be correlated with the individual measurement error that remains in the equation of interest (i.e., insurance status). This is due to the fact that in states with a relatively low-income eligibility threshold, the expected individual measurement error is also small since most persons in that state will have incomes above the threshold. Furthermore, the correlation between the average measurement error in the national sample and the measurement error of the individual may be particularly strong because Cutler and Gruber (1996) use a state-fixed effects model that essentially uses only 
within-state variation in Medicaid eligibility thresholds. ${ }^{2}$ It is precisely the differences in eligibility thresholds that generate the correlation in the first place, and the correlation is expected to be stronger within-states than across states because of state differences in income distributions. The upshot is that "simulated eligibility" may not be a valid instrument since it is correlated with the error of the equation of interest. Estimates obtained using this instrument may be biased. ${ }^{3}$

A more recent study of crowd out is by Shore-Sheppard (1997) who uses aggregate data to estimate the extent of crowd out among children. In this study, Shore-Sheppard (1997) aggregates individual level data from the CPS to the state, age and income level and regresses changes in insurance coverage between 1988 and 1993 on changes in the fraction eligible between these two time points (i.e., a state fixed-effects model). Shore-Sheppard (1997) concluded that the extent of crowd out of private insurance is approximately 15 percent. Shore-Sheppard (1997) argued that the difference between her study and that of Cutler and Gruber (1996) stemmed from the fact that she was "... measuring the impact of the expansions on the newly eligible..." (ShoreSheppard 1997, p. 28). In contrast, she argued that Cutler and Gruber (1996) measured the impact of the expansions on the always eligible and the newly eligible. In fact, the model Shore-Sheppard (1997) estimated is quite similar to the model that Cutler and Gruber (1996) estimated, and both measured the effect of the expansions on the same groups.

Both studies estimated state fixed-effects models with the only difference being that Shore-Sheppard

\footnotetext{
${ }^{2}$ We abstract from their use of within-state variation in eligibility that comes from differences in child age.

${ }^{3}$ The bias can be formally shown as follows. Let the equation of interest (e.g., private insurance) be (1) $P_{i j t}=\alpha_{0}+\alpha_{1} E^{*} i j t+v_{i j t}$
} where $P$ is insurance status and $E^{*}$ is true Medicaid eligibility. Eligibility is measured with error so the estimated equation is (2) $P_{i j t}=\alpha_{0}+\alpha_{1} E_{i j t}-\alpha_{1} w_{i j t}+v_{i j t}$

In equation (2), true eligibility has been replaced by measured eligibility. Cutler and Gruber (1996) instrument for measured eligibility in equation (2) using what they refer to as simulated eligibility, which is which is the average eligibility of a national sample of children determined by applying that state's eligibility rules to the national sample. The instrumental variables estimator is in deviations form

(3) $\alpha_{1}=\sum\left(Z_{j t}-u_{j t}\right) P_{i j t} / \sum\left(Z_{j t}-u_{j t}\right) E_{i j t}$ where $Z_{j t}$ is the simulated eligibility measure (true eligibility plus error). If we substitute for $P$ in equation (3), we get (4) $\alpha_{1}=\sum\left(Z_{j t}-u_{j t}\right)\left(\alpha_{1} E_{i j t}-\alpha_{1} w_{i j t}+v_{i j t}\right) / \sum\left(Z_{j t}-u_{j t}\right) E_{i j t}$ 
aggregated the data to the state level. Thus, both studies may find a significant relationship between insurance status and Medicaid eligibility even if eligibility did not change-i.e., if there were in fact no newly eligible children. The reason for this result is that the take-up rates (i.e., the coefficient on eligibility) for Medicaid and private insurance may change over time, a point Shore-Sheppard (1997) makes other places in the paper. In fact, we present evidence later in the paper that shows this is in fact the case for Medicaid. Thus, differences in the two studies' findings must result from some other difference between the studies. In fact, when Shore-Sheppard (1997) estimated a model using individual-level data, she found a crowd out effect of 31 percent (Table 8 of Shore-Sheppard 1997), exactly what Cutler and Gruber (1996) found (Table IV of Cutler and Gruber 1996). Thus, it is the aggregation of the data that accounts for the differences in results between Shore-Sheppard (1997) and Cutler and Gruber (1996). One consequence of aggregation is that Shore-Sheppard (1997) is unable to include many control variables that are correlated with insurance status and Medicaid eligibility. For example, race, family composition and the number of employed individuals in the family are all significant determinants of insurance status that are also correlated with Medicaid eligibility, and the error in eligibility. These variables are all omitted from the aggregate analysis of Shore-Sheppard (1997), and differences in these variables at the statelevel may have confounded her estimates that use such data. Moreover, the aggregate fixed-effects model she estimates may have exacerbated measurement error problems that are potentially serious in cross-sectional settings, but which may be worsened by taking first-differences of the data.

A different type of analysis of crowd out was presented by Dubay and Kenney (1996a). In this study, Dubay and Kenney (1996a) used the CPS to examine changes in insurance coverage between 1988 and 1993 for two groups of children: children from families with incomes under 100 percent of poverty and children from families with incomes between 100 and 133 percent of poverty. These two groups of children represent the target groups of the Medicaid eligibility expansions. The authors also examined changes in insurance coverage of males ages 18 to 44 in the relevant income categories to control for time variation in insurance coverage during this period. To estimate the impact of the Medicaid eligibility expansions on insurance coverage, Dubay and Kenney

The bias in $\alpha_{1}$ stems from the correlation between $u_{j t}$ and $w_{i j t}$. 
(1996a) subtract the changes in insurance coverage for men from the changes in insurance coverage for children. Based on these difference-in-differences calculations, Dubay and Kenney (1996a) concluded that between 15 and 22 percent of enrollment in Medicaid came from private insurance, an effect similar to that obtained by ShoreSheppard (1997).

There are two aspects of the Dubay and Kenney (1996a) study that merit remarks. First, the use of men as a control group may be problematic since time variation in insurance coverage of men may differ from that of children. Second, if measurement error associated with family income resulted in men and children being misclassified as poor or near poor, then the difference-in-differences estimates that Dubay and Kenney obtain will be downward biased. We take up this point later in the analysis when we describe our methodology, which is similar to that of Dubay and Kenney (1996a).

Our review of the literature has highlighted two points. First, previous studies have not reached a consensus on the extent of crowd out and additional study is warranted. Second, measurement error associated with Medicaid eligibility and a lack of appropriate controls for state and time variation in insurance status may have confounded previous regression based estimates of crowd out. In light of these issues, we undertake an analysis of crowd out using longitudinal data and a difference-in-differences methodology. We believe that our approach addresses some of the problems that affected previous studies and provides credible alternative estimates of crowd out.

\section{Research Strategy}

One strategy to estimate the effects of Medicaid eligibility expansions on insurance status using longitudinal data is to form treatment and control groups based on changes in Medicaid eligibility, and to compare changes in insurance status over time between the two groups. This is sometimes referred to as a difference-indifferences (DD) analysis. An advantage of the DD analysis is that it obtains estimates of the extent of crowd out by examining changes in insurance status that are directly linked to changes in eligibility, something previous studies were unable to do. In our application of the DD procedure, the treatment group consists of children who 
gained eligibility as a result of the eligibility expansions, and the control group consists of children who were either always eligible or never eligible for Medicaid before and after the expansions.

A critical assumption underlying the DD analysis is that unmeasured, time-varying factors that affect insurance status have the same effect on treatment and control group members. If this is not the case, then DD estimates are biased. This problem is not unique to the DD analysis: all non-experimental studies require controls for time-varying factors that affect the outcome of interest. For example, Cutler and Gruber (1996) use year dummy variables to control for time trends in insurance status. As noted previously, this strategy may result in biased estimates if time variation in children's insurance coverage differs by eligibility status, which is also time varying. In fact, we present evidence later in the paper that shows that time variation in insurance coverage differs by eligibility status. We address this problem by selecting only a portion of the children not affected by the Medicaid expansions to serve as controls for children who were affected. We select that group of unaffected children who experienced similar life events (e.g., divorce) that affect children's insurance coverage, as that experienced by the group of children affected by the Medicaid expansions.

Another potential problem with our simple DD analysis is that measurement error in Medicaid eligibility may lead to contamination of our treatment and control groups. We use the phrase contamination to refer to the case in which some members of the treatment group are unaffected by the Medicaid expansions and some members of the control group are affected by the expansions. In our case, misclassification of treatments and controls may be significant because of the difficulty of correctly assigning Medicaid eligibility. The consequence of misclassification is that DD estimates will be biased. If the misclassification is relatively minor, DD estimates will be biased downward, but if it is extreme, DD estimates may even have the wrong sign. We can demonstrate this point as follows. Let the true estimate of the effect of the expansions on insurance status be (1) $\mathrm{DD}=(\mathrm{B}-\mathrm{A})-(\mathrm{D}-\mathrm{C})$,

where $\mathrm{A}$ is the mean level of private insurance for the true treatment group before the expansions, $\mathrm{B}$ is the mean level of private insurance for the true treatment group after the expansions, and C and D are similar quantities for the control group. If we assume that there is misclassification only among the treatment group and that in fact 50 percent of the observed treatment group consists of members of the control group, then the DD estimate becomes 


$$
\mathrm{DD}^{\prime}=.5 \mathrm{DD}=.5[(\mathrm{~B}-\mathrm{A})-(\mathrm{D}-\mathrm{C})]=(.5 \mathrm{~B}+.5 \mathrm{D})-(.5 \mathrm{~A}+.5 \mathrm{C})-(\mathrm{D}-\mathrm{C})
$$

The DD' estimate is 50 percent of the true DD estimate. Similarly, if 30 percent of the treatment group consists of controls, then the DD' estimate is 70 percent of the true DD estimate.

The upshot is that contamination of the treatment group can seriously bias our estimates, but note that we know the direction of the bias. As long as there is no misclassification in the control group, the DD' estimate will have the same sign as the true DD estimate. In fact, only if the sum of the proportion of children misclassified in the treatment and control groups is greater than one will our DD estimate have the wrong sign. We believe this to be a very unlikely occurrence and the data we present later supports this claim. More importantly, the magnitude of the bias in the DD estimates will be the same regardless of whether the outcome of interest is private insurance or Medicaid coverage since we use the same treatment and control group in each case. Therefore, the misclassification bias is the same for both outcomes. This implies that our estimate of crowd out will be unbiased since the estimate of crowd out is formed by taking the ratio of the two DD estimates-one for the effect of the expansions on private insurance and one for the effect of the expansions on Medicaid. ${ }^{4}$

One solution to the contamination problem is to choose treatment and control groups that are the least likely to be contaminated by misclassification. In our application, children from families that were never eligible for Medicaid may be a good candidate to be a control group since their families are located relatively high in the income distribution where the measurement error and misclassification problem is least likely to be important. The disadvantage of this choice, however, is that time variation in insurance status for this never-eligible group may be quite different from the time variation of the newly eligible group of children. This would render the never eligible group an inappropriate control group even though it is a group relatively free from contamination bias. The other choice of control group is to use poor, non-AFDC children who were always eligible for Medicaid. While this group of children may have similar trends in insurance status, they are more likely to be incorrectly classified.

\footnotetext{
${ }^{4}$ This point can be easily demonstrated. Let $\mathrm{DD}_{\mathrm{p}}$ represent the true estimate of the effect of the eligibility expansions on private insurance, and $\mathrm{DD}_{\mathrm{m}}$ represent the true estimate of the effect of the eligibility expansions on Medicaid. Taking the absolute value of the ratio of $\mathrm{DD}_{\mathrm{p}}$ to $\mathrm{DD}_{\mathrm{m}}$ forms an estimate of crowd out. Our problem is that instead of $\mathrm{DD}_{\mathrm{p}}$, we obtain
} 
It is important to note how the measurement error problem associated with Medicaid eligibility manifests itself differently in the DD analysis than in the regression analyses of previous researchers. In the latter case, measurement error resulted in biased estimates of indeterminate sign. In our case, measurement error results in downward biased DD estimates and potentially unbiased estimates of crowd out.

\section{Data}

The data used in this analysis come from the 1988 and 1992 waves of the National Longitudinal Survey of Youth (NLSY). These data provide a unique opportunity to examine the effects of Medicaid expansions on changes in health insurance coverage of poor and near-poor children since we can follow the same child over time. The NLSY is a longitudinal survey of youth that were born between the years 1957 and $1965 .^{5}$ An important aspect of the NLSY for our purposes is that it provides extensive information about the children of all female respondents. We focus on a sample of children who were less than eight years old in 1988 and who are between the ages of 4 and 12 by 1992. We do not include older children because the mothers of older children had their first birth at relatively young ages, which raises questions about the representativeness of these children and women.

The NLSY contains information on the health insurance coverage of each child. The insurance status of the child comes from mother's reports and the survey question allows for only three responses: private insurance, Medicaid, or no insurance. Those children covered by other types of insurance such as CHAMPUS are in most cases included in the private category. ${ }^{6}$ In addition, the NLSY includes extensive information on demographic

\footnotetext{
$.5 \mathrm{DD}_{\mathrm{p}}$, and instead of $\mathrm{DD}_{\mathrm{m}}$, we obtain $.5 \mathrm{DD}_{\mathrm{m}}$. The ratio of the two biased estimates, however, is equal to the ratio of the two unbiased estimates. Thus, we obtain an unbiased estimate of crowd out.

${ }^{5}$ The NLSY oversamples black, Hispanic and economically disadvantaged white youths. Since the analysis uses a constant population of children-i.e., those present in both 1988 and 1992, the oversampling of economically disadvantaged white youth does not present a problem, as these respondents are excluded from the analysis since they were dropped from the survey in 1991.

${ }^{6}$ In the 1992 survey, the mother was also asked about the source of her children's health insurance. This question allowed for more detailed responses than the question we used to determine insurance status, but it was not child specific. A crosstabulation of the mother's response to this question showed that over 80 percent of the mothers who responded "other" to the detailed question responded "private" to the less detailed question. Less than four percent of the sample has other types of insurance.
} 
characteristics of the mother such as age, marital status, education, income, and employment that we use in the analysis. We assigned Medicaid eligibility using family wage income in the past-calendar year. ${ }^{7}$

We impose two other restrictions on the sample. First, we drop from the sample children of mothers who had 16 or more years of education by 1988 . Children from these families are in the vast majority of cases not eligible for Medicaid and time variation in health insurance coverage for this group of children may be very different than for children from other types of families, particularly those low-income children affected by the Medicaid expansions. By eliminating these families from the sample, we reduce the unmeasured heterogeneity in the sample that may confound our estimates, and make it more likely that our control group will be

appropriate. Second, we drop from the analysis children from families receiving AFDC payments at the time of the 1988 interview. Again, it is our view that children from these families will experience different changes in health insurance status than children from other types of families, and that by eliminating them we reduce the unmeasured heterogeneity in the sample. In fact, the opportunity set for these children is fundamentally different from the opportunity set for other children since all children on AFDC are enrolled in Medicaid and thus the only changes in insurance status that could be observed is movement to private insurance or uninsured.

\section{Empirical Findings}

\section{A. Regression Analysis of Insurance Status on Medicaid Eligibility}

To link our analysis with previous studies, we estimated regression models similar to those estimated by Cutler and Gruber (1996) and Shore-Sheppard (1997). We report these estimates in Table 1. The first models we present used pooled cross-sectional data and estimates from these models are listed in the top panel of Table 1. We formed two cross-sections of children less than nine years old in 1988 and 1992 . We estimated linear probability models of insurance status controlling for Medicaid eligibility status and a variety of other characteristics that are listed in the notes to Table 1 . The specification is very similar to that used by Cutler and

\footnotetext{
${ }^{7}$ We also experimented with total family income in the past year and current wage income. It made little difference in the results relevant to Table 1 which measure of income was used to calculate eligibility.
} 
Gruber (1996) and Shore-Sheppard (1997). Our sample of children are younger, however, as these two previous studies used children up to age fifteen, and we have restricted the sample to non-AFDC recipients and to children of mothers with less than 16 years of education.

We present ordinary least squares estimates and instrumental variables (IV) estimates. We instrument for eligibility to control for behavioral changes that are associated with changes in Medicaid income eligibility thresholds. For example, women may decrease their labor supply in order to qualify for the higher Medicaid eligibility threshold. While this behavior is a consequence of the eligibility expansions, there is some theoretical interest in obtaining estimates of what Moffitt (1992) refers to as the "mechanical" impact of changes in program attributes. In this case, the "mechanical" impact would be the effect of the eligibility expansions that comes from making families with incomes previously above the old threshold, but below the new threshold, eligible.

Accordingly, we expect the IV estimates to be smaller than the OLS estimates since the latter measures the "mechanical" and "behavioral" impact of the Medicaid expansions. We instrument for Medicaid eligibility using eligibility calculated with a constant (1988) income. This is similar to the procedure used by Shore-Sheppard (1997). ${ }^{8}$

As shown in Table 1, our estimates indicate that Medicaid eligibility has a relatively large negative effect on private insurance status, and a relatively small positive effect on Medicaid participation. All estimates are statistically significant. These effects differ from those reported by previous research. For example, Cutler and Gruber (1996) report estimates of the effect of Medicaid eligibility of -0.074 and 0.235 on private insurance and Medicaid respectively. An estimate of crowd out can be obtained by forming the ratio of the estimates presented in columns 1 and 2 of Table 1. Doing this calculation implies crowd out of between 147 and 183 percent. Taken at face value, the estimates suggest that the eligibility expansions caused private insurance to go down so much that the increased enrollment in Medicaid was insufficient to offset the loss in insurance. As a result, the number of uninsured increased. This anomalous result raises questions about the credibility of these regression estimates and suggests that the Medicaid eligibility coefficient may be capturing some unmeasured time variation in 
insurance coverage. It is also interesting to note that the instrumental variables (IV) estimate of crowd out is larger than the OLS estimate. This is also unexpected since the IV estimates are intended to measure the "mechanical" impact of the Medicaid expansions, and are expected to be smaller than the OLS estimates.

We also estimated fixed-effects models since we had two observations for each child. We report estimates from these models in the lower panel of Table 1. These estimates are obtained using a constant sample of children who were less than nine years old in 1988 and between the ages of 4 and 12 in 1992 . We estimated the fixed-effects models a number of ways including use of Chamberlain's (1980) conditional logit procedure and by using instrumental variables. All estimates in the bottom panel of Table 1 indicate crowd out of at least 100 percent since the absolute value of the estimate of the effect of Medicaid eligibility on private insurance is larger, or approximately equal to, the estimate of the effect of Medicaid eligibility on Medicaid enrollment. In addition, the IV estimate of crowd out is again larger than the OLS estimate.

Estimates of crowd out derived from Table 1 greatly exceed those in the literature. This result may be due to a variety of factors including different samples, different measures of income used to determine Medicaid eligibility, differences in the time period of analysis and slight differences in model specification. Our interest in these estimates, however, is mainly as a point of reference for the results from our difference-in-differences analysis. We are interested in comparing these regression estimates, obtained using methods similar to other researchers, to the difference-in-differences estimates we present below. Remember, that the measurement error problem associated with Medicaid eligibility affects the two types of analyses differently, and the two analyses use different methods to control for time variation insurance coverage. A comparison of the two sets of estimates may provide insight into the severity of these empirical problems in previous analyses.

\section{B. Distribution of Health Insurance Coverage by Medicaid Eligibility Status - 1988 to 1992}

In Table 2, we show the distribution of children's health insurance status in 1988 and 1992 by Medicaid eligibility status. We have defined five groups of children based on their eligibility for Medicaid in 1988 and

\footnotetext{
${ }^{8}$ Shore-Sheppard (1997) reported on sensitivity tests that investigated differences in estimates between her paper and Cutler and Gruber (1996) due to differences in instrument choice. She concluded that the different instrument choice explained
} 
1992. Our definitions attempt to identify children that were affected by the expansions, treatment groups, and children unaffected by the expansions, control groups. For example, children who were not eligible for Medicaid in 1988 or 1992 represent a potential control group since they were never eligible for Medicaid and should not have been affected by the eligibility expansions. On the other hand, children who gained eligibility between 1988 and 1992 represent a treatment group. The top panel of Table 2 shows the distribution of health insurance status in 1988 and the middle panel shows similar figures for 1992. The bottom panel shows changes in insurance status by the various groups.

For our purposes, the bottom panel of Table 2 contains the interesting results. The figures in the bottom panel indicate that there was significant growth in Medicaid coverage between 1988 and 1992. Children who were always eligible for Medicaid increased their coverage by 25 percentage points and newly eligible children increased their coverage by between 17.3 to 30.0 percentage points depending on the reason for their new eligibility. Children whose eligibility gain was not due to a decrease in family income increased enrollment less than children whose eligibility gain was due to a decrease in family income. It is surprising that the increases in Medicaid enrollment were so similar among the always eligible and newly eligible children. Intuition suggests that Medicaid coverage would have increased more among newly eligible children than among children who were always eligible for Medicaid. One explanation for this finding is that other aspects of the eligibility expansions such as changes in enrollment procedures and increases in the number of participating physicians may have caused a significant increase in take-up among the eligible population. Another explanation is that eligibility is measured with some error and that a portion of children in the always-eligible group actually gained eligibility. ${ }^{9}$

There are two other results of interest shown in the bottom panel of Table 1. First, there was a substantial decrease in the number of uninsured children, particularly among children from families who were always eligible for Medicaid. Again, this is a surprising finding. Why didn't these uninsured children participate in Medicaid in 1988? Perhaps it was because in 1988 Medicaid enrollment procedures were more onerous, but it may also be

virtually none of the differences in the estimates.

${ }^{9}$ Clearly, there is measurement error in eligibility, although there is no definitive way to measure the true extent of the problem. For example, in 1988, 4.3 percent of the children in the two "gained eligibility" categories report Medicaid coverage. 
related to measurement error in eligibility. Second, there was a significant decrease in private insurance among children in the group that "gained eligibility" due to a loss of family income. This could naively be interpreted as evidence of crowd out, but this inference ignores any trend in private insurance.

Estimates of the effect of the Medicaid expansions on insurance coverage can be formed by taking differences of the changes in insurance coverage of our treatment and control groups. We begin with what we consider to be the first treatment group: always eligible children. We consider this a treatment group because changes in Medicaid enrollment procedures and physician supply that resulted from the eligibility expansions may have affected this group of children. We compare changes in insurance status of this group to changes in insurance status of the never eligible group whose family had no loss of income. The difference-in-differences estimates indicate an insignificant decrease in private insurance of $1.4(-1.0-0.4)$ percentage points, and a significant increase in Medicaid enrollment of $26.2(25.0+1.2)$ percentage points. It is important to note that these DD estimates may be downward biased because of misclassification and that an estimate that is not statistically significant may simply be one that is biased toward zero. Therefore, it is important to form the ratio of the two potentially biased estimates to obtain an unbiased estimate of crowd out. In this case, the DD estimates indicate crowd out of $5.3(-1.4$ / 26.2) percent. Note that the crowd out estimate is a percent. It represents the percent of Medicaid enrollment that came from private insurance.

The second treatment group is children that became eligible solely because of increases in the income eligibility threshold. In other words, these children's family incomes stayed relatively constant, but because of the higher income eligibility threshold they were made eligible for Medicaid. For this treatment group, we again we use children who were never eligible and whose family did not suffer an income loss as a control group. In this case we observe an insignificant 2.6 percentage point decrease in private insurance and a significant 18.5 percentage point increase in Medicaid enrollment. Taking the ratio of these two estimates yields an estimate of crowd out of 14.1 percent.

The last treatment group is children who became eligible because of changes in family income. In this group, 47 percent of the families experienced a divorce. Given the changes in family circumstance, we use a group of never eligible children from families who also suffered a loss of income as controls. Approximately 26 
percent of these families experienced a divorce. For this treatment group, we obtain a significant 10.1 percentage decrease in private insurance coverage and a significant 27.1 percentage point increase in Medicaid enrollment. This implies a crowd out effect of 37.3 percentage points. We also experimented with a slightly different control group. Instead of using all never eligible children whose families suffered a loss of income, we restricted it to families whose income in 1988 was below $\$ 40,000$. This alternative control group experienced a 15.8 percentage point decrease in private insurance and a 4.0 percentage point increase in Medicaid enrollment between 1988 and 1992. Using these changes in insurance coverage to obtain estimates of crowd out yields a crowd out effect of 23.9 (6.2 / 26) percentage points. This last calculation illustrates the critical role that the control group plays in the difference-in-differences analysis. While we believe that never eligible children whose family suffered an income loss are a better control group than never eligible children whose family had no loss of income, we are still not certain that they are a perfect control group. When we selected never eligible families in the lower part of the income distribution for this group we reduced our estimate of crowd out. Based on this we believe the 37.3 percentage point estimate of crowd out to be an upper bound, and prefer to emphasize the 23.9 percent estimate.

\section{Discussion}

This study analyzed the effect of Medicaid eligibility expansions on health insurance coverage for children using the National Longitudinal Survey of Youth (NLSY). Our study differed from previous studies in two significant ways. First, we used longitudinal data that allowed us to directly relate changes in eligibility status to changes in insurance status. Second, we did not use a regression-based approach, but instead used a difference-in-differences (DD) analysis. The advantages of the DD analysis is that it is potentially easier to address the measurement error associated with Medicaid eligibility and it incorporates potentially better controls for time variation in insurance coverage.

Our results suggest that the Medicaid expansions resulted in relatively little crowd out of private insurance. In our sample we obtained estimates of crowd out between 5.3 and 23.9 percent depending on the eligibility status of the child. If we combine these estimates, we obtain an estimate of crowd out of 14.5 percent. 
Thus, 14.5 percent of the increased enrollment into Medicaid came from private insurance. This is similar to the effects reported by Shore-Sheppard (1997) and Dubay and Kenney (1996a).

A notable finding of our study is the large difference in crowd out estimates obtained using different statistical methodologies. The regression-based approach yielded extremely large estimates of crowd out while the difference-in-differences (DD) analysis found relatively little crowd out. The regression estimates may be biased because of measurement error and because of potentially inadequate controls for time variation in insurance coverage. The difference-in-differences approach may also be biased for the same reasons. Which estimate is correct? Before discussing potential answers to this question, we note that the data in Table 2 appear to be inconsistent with the magnitude of the regression-based estimates of crowd out. Our inspection of this data leads us to conclude that the regression estimates are simply too large to be credible. In addition, the regressionbased estimates were not internally consistent. The loss of private insurance associated with the Medicaid expansions was so great that it actually increased the number of uninsured. This suggests that the estimates were measuring some trend in the data not captured by other variables.

Although common empirical problems plague the regression and DD procedures, there is a difference as to how these problems manifest themselves. Measurement error leads to a downward biased DD estimate of the effect of the eligibility expansions on insurance status, but a potentially unbiased estimate of crowd out. On the other hand, measurement error leads to a bias of indeterminate sign in the regression approach. Second, the lack of adequate controls potentially plagues both analyses. In the DD analysis, however, we had flexibility in defining control groups and formed appropriate control groups for different treatment groups. For example, it would have been incorrect to use never eligible children whose family suffered no loss of income to control for time variation in insurance coverage of children who gained eligibility due to a loss of family income. In contrast, the regression approach assumes common time trends for both eligible and ineligible children.

While we cannot provide a definitive answer to which estimate of crowd out is correct, we believe that the DD estimate of crowd out is much more consistent with the data than the estimate of crowd out obtained using regression methods. We also feel that the measurement error problem is more problematic in the regression approach than in the DD analysis. Based on these considerations we conclude that crowd out is a relatively minor 
problem. The implication of this finding is that recent expansions in eligibility for publicly provided health insurance will be effective at reducing the number of uninsured children. 


\section{References}

Acs, G., and C.E. Steuerle. 1993. "Trends in the Distribution of Non-Wage Benefits and Total Compensation." Urban Institute Working Paper. Washington D.C.: Urban Institute.

Congressional Research Service. 1988. "Medicaid Source Book: Background Data and Analysis." Washington, D.C.: U.S. Government Printing Office. . 1993. "Medicaid Source Book: Background Data and Analysis (A 1993 Update)." Washington, D.C.: U.S. Government Printing Office.

Cutler, D.M., and J. Gruber. 1996. “Does Public Insurance Crowd Out Private Insurance?” Quarterly Journal of Economics 111: 391-430.

Dubay, L., and G. Kenney. 1996a. "The Effects of Medicaid Expansions on Insurance Coverage of Children” The Future of children 6(1): 152-161.

Dubay, L., and G. Kenney. 1996b. "Did the Medicaid Expansions For Pregnant Women Crowd Out Private Coverage?” Washington D.C.: The Urban Institute. Unpublished Manuscript.

Fronstin, P. 1996. “The Decline in Health Insurance and Labor Market Trends.” Statistical Bulletin JulySeptember: 28-36.

Hill, I.T. 1992. "The Medicaid Expansions for Pregnant Women and Children: A State Program Characteristics Information Base." Prepared for the Office of Research, Health Care Financing Administration: U.S. Department of Health and Human Services.

Moffitt, Robert. 1992. "Incentive Effects of the U.S. Welfare System.” The Journal of Economic Literature, 30:1-61.

Monheit, A.C., and C.L. Schur. 1988. “The Dynamics of Health Insurance Loss: A Tale of Two Cohorts.” Inquiry 25: $315-327$.

Newacheck, P.W., Hughes, D.C., and M. Cisternas. 1995. "Children and Health Insurance: An Overview of Health Affairs 2: 244-254.

Shore-Sheppard, L.D. 1995. "Stemming the Tide? The Effect of Expanding Medicaid Eligibility on Health Princeton University Industrial Relations Section Working Paper \#361.

Shore-Sheppard, L.D. 1997. "Stemming the Tide? The Effect of Expanding Medicaid Eligibility on Health Insurance Coverage.” Unpublished paper University of Pittsburgh.

U.S. Committee on Ways and Means. 1991. Green Book: Background Material and Data on Programs Within the Jurisdiction of the Committee on Ways and Means. Washington, D.C.: U.S. Government Printing Office. 
Table 1

Estimates of the Effect of Medicaid Eligibility on Insurance Status

\begin{tabular}{|l|c|c|c|}
\hline Cross-Sectional Estimates (N=5482) & $\begin{array}{c}\text { Probability of } \\
\text { Private Coverage }\end{array}$ & $\begin{array}{c}\text { Probability of } \\
\text { Medicaid Coverage }\end{array}$ & No Insurance \\
OLS & & & \\
& & & \\
& & & \\
Instrumental Variables (IV) & $(0.014)$ & $(0.161$ & $0.013)$ \\
& -0.219 & 0.120 & 0.128 \\
Fixed-Effect Estimates (N=2244) & $(0.021)$ & $(0.013)$ & $(0.195)$ \\
OLS & & & \\
& & & 0.043 \\
Instrumental Variables (IV) & -0.137 & 0.140 & $(0.021)$ \\
& $(0.020)$ & $(0.015)$ & 0.160 \\
Chamberlain's Conditional Logit & -0.166 & 0.031 & $(0.026)$ \\
[Marginal Effect in Brackets] & $(0.025)$ & $(0.019)$ & 0.416 \\
& -1.0635 & 1.665 & $(0.192)$ \\
\hline
\end{tabular}

Notes: Cross-sectional models include the following variables: race (3 dummy variables), sex and age (5 dummy variables) of child; mother's age (quadratic), education (3 dummy variables), labor force participation status and marital status; number of children in family, region ( 9 dummy variables); year dummy, and region by year dummy variables. The instrument used in the IV models is Medicaid eligibility status determined using 1988 wage income. Fixed-effects models include the following variables: mother's marital status, labor force participation and number of children. Standard errors are in parentheses. 
Table 2

Insurance Status By Medicaid Eligibility Status Constant Population of Children Ages 0 to 8 in 1988 and Ages 4 to 12 in 1992

\begin{tabular}{|c|c|c|c|}
\hline & & 1988 Insurance Status & \\
\hline & $\begin{array}{l}\text { Private Coverage } \\
(\%)\end{array}$ & $\begin{array}{l}\text { Medicaid Coverage } \\
(\%)\end{array}$ & $\begin{array}{l}\text { Uninsured } \\
(\%)\end{array}$ \\
\hline $\begin{array}{l}\text { Always Eligible }(\mathrm{N}=319) \\
\text { Eligible in } 1988 \text { and } 1992\end{array}$ & 43.6 & 17.6 & 40.1 \\
\hline $\begin{array}{l}\text { Gained Eligibility Due to Expansions }(\mathrm{N}=92) \\
\text { Not Eligible in 1988, Eligible in } 1992\end{array}$ & 56.5 & 9.8 & 33.7 \\
\hline $\begin{array}{l}\text { Gained Eligibility Due to Loss in Income (N=260) } \\
\text { Not Eligible in 1988, Eligible in } 1992\end{array}$ & 70.8 & 2.3 & 26.9 \\
\hline $\begin{array}{l}\text { Never Eligible No Loss of Income (N=1131) } \\
\text { Not Eligible in } 1988 \text { and } 1992\end{array}$ & 88.3 & 0.6 & 11.1 \\
\hline Never Eligible Loss of Income ( $\mathrm{N}=243)$ & 90.9 & 1.2 & 7.8 \\
\hline & & 1992 Insurance Status & \\
\hline & $\begin{array}{c}\text { Private Coverage } \\
(\%)\end{array}$ & $\begin{array}{c}\text { Medicaid Coverage } \\
(\%)\end{array}$ & $\begin{array}{l}\text { Uninsured } \\
(\%)\end{array}$ \\
\hline $\begin{array}{l}\text { Always Eligible }(\mathrm{N}=319) \\
\text { Eligible in } 1988 \text { and } 1992\end{array}$ & 42.6 & 42.6 & 21.0 \\
\hline $\begin{array}{l}\text { Gained Eligibility Due to Expansions (N=92) } \\
\text { Not Eligible in 1988, Eligible in } 1992\end{array}$ & 54.3 & 27.2 & 25.0 \\
\hline $\begin{array}{l}\text { Gained Eligibility Due to Loss in Income }(\mathrm{N}=260) \\
\text { Not Eligible in 1988, Eligible in } 1992\end{array}$ & 48.8 & 32.3 & 23.8 \\
\hline $\begin{array}{l}\text { Never Eligible No Loss of Income (N=1131) } \\
\text { Not Eligible in } 1988 \text { and } 1992\end{array}$ & 88.7 & 1.3 & 10.0 \\
\hline Never Eligible Loss of Income ( $\mathrm{N}=243)$ & 79.0 & 4.1 & 16.9 \\
\hline & & 1992-1988 & \\
\hline & $\begin{array}{c}\text { Private Coverage } \\
(\%)\end{array}$ & $\begin{array}{l}\text { Medicaid Coverage } \\
(\%)\end{array}$ & $\begin{array}{c}\text { Uninsured } \\
(\%)\end{array}$ \\
\hline $\begin{array}{l}\text { Always Eligible }(\mathrm{N}=319) \\
\text { Eligible in } 1988 \text { and } 1992\end{array}$ & -1.0 & $25.0 *$ & $-19.1 *$ \\
\hline $\begin{array}{l}\text { Gained Eligibility Due to Expansions (N=92) } \\
\text { Not Eligible in 1988, Eligible in } 1992\end{array}$ & -2.2 & $17.3 *$ & -8.7 \\
\hline $\begin{array}{l}\text { Gained Eligibility Due to Loss in Income ( } \mathrm{N}=260) \\
\text { Not Eligible in 1988, Eligible in } 1992\end{array}$ & $-22.0 *$ & $30.0 *$ & -3.1 \\
\hline $\begin{array}{l}\text { Never Eligible No Loss of Income }(\mathrm{N}=1131) \\
\text { Not Eligible in } 1988 \text { and } 1992\end{array}$ & 0.4 & -1.2 & -1.1 \\
\hline $\begin{array}{l}\text { Never Eligible Loss of Income }(\mathrm{N}=243) \\
\text { Not Eligible in } 1988 \text { and } 1992\end{array}$ & $-11.9 *$ & 2.9 & $9.1 *$ \\
\hline
\end{tabular}

Note: The total sample size is 2244 , the same as that used in the fixed effects regressions in Table 1. Those children who lost eligibility are omitted from this table. All figures are percentages. Rows do not sum to one because some mother's reported dual insurance coverage or their children. Eligibility is determined using year-specific income and eligibility rules. An asterisk next to a number indicates a statistically significant $(\mathrm{p}<.05)$ difference. 